\title{
Multidisciplinary treatment approach of a patient with amelogenesis imperfecta: A case report
}

\author{
Jihan M. Turkistani ${ }^{1}$, Abdullah S. Almushayt ${ }^{1}$, Sami A. Farsi ${ }^{2}$, Jihan M. Turkistani ${ }^{3 *}$ \\ ${ }^{1}$ Faculty of Dentistry, King Abdulaziz University, Jeddah, Saudi Arabia \\ ${ }^{2}$ King Fahad General Hospital, Jeddah, Saudi Arabia \\ ${ }^{3}$ King Abdulaziz Medical City-Jeddah, Jeddah, Saudi Arabia \\ Email: dr_jihant@hotmail.com \\ Received 3 July 2013; revised 4 August 2013; accepted 1 September 2013 \\ Copyright (C) 2013 Jihan M. Turkistani et al. This is an open access article distributed under the Creative Commons Attribution Li- \\ cense, which permits unrestricted use, distribution, and reproduction in any medium, provided the original work is properly cited.
}

\section{ABSTRACT}

Introduction: Amelogenesisimperfecta (AI) is a developmental disturbance, genomic in origin, which interferes with normal enamel formation of both primary and permanent dentitions in the absence of a systemic disorder. Three major categories can be recognized clinically, namely hypoplastic, hypomaturation and hypocalcified. Timely and comprehensive intervention is critical to spare the patient from psychological consequences of these disfiguring conditions. Clinical Report: A 10-year-old boy was presented with dissatisfaction with appearance of his teeth sensitivity and poor masticatory efficiency. His medical history was noncontributory. Composite restorative material was selected as a suitable replacement of the defective structures because of its esthetics and high sustainability. Treatment Objectives: The multidisciplinary approach was oriented toward achieving functional and esthetic rehabilitation of these teeth with minimal chair-side time. Treatment Outcomes: The use of composite restorative material resulted in successful and satisfactory enhancement of the patient's esthetic appearance, as well as protection against further wear and sensitivity thereby improving his functional demands. The longevity of the treatment outcome required meticulous maintenance of oral hygiene and patient compliance. Conclusion: Management of a patient with AI is a challenge for the clinician. Treatment options vary considerably depending on several factors such as age of the patient, socioeconomic status, severity of the disorder, and most importantly, the patient's cooperation. Composite restorative material is considered an excellent conservative transitional treatment for protec-

"Corresponding author. tion of AI weakened teeth.

Keywords: Amelogenesis Imperfecta; Hypocalcified Enamel; Management; Multidisciplinary; Composite Restorative Material

\section{CASE PRESENTATION}

A healthy, 10-year-old Saudi boy was referred to the pediatric dentistry specialty clinic at King Abdulaziz University by a general dental practitioner for treatment of his disfigured teeth. His chief complaints were sensitivity to hot and cold, dissatisfaction with the appearance of his teeth, and a compromised masticatory function. The medical history indicated no contraindications for dental treatment.

A thorough extra and intraoral clinical evaluation revealed poor oral hygiene with plaque accumulation, which had resulted in generalized gingivitis. The patient presented short clinical crowns with generalized areas of thin, discolored, hypoplastic enamel and areas of fractured enamel and exposed dentin (Figure 1).

A premature loss of vertical dimension, as well as an overbite of $30 \%$ and an over jet of $6 \mathrm{~mm}$, was evident. A Class II Angle molar relationship, division I malocclusion, was evidenced on both right and left sides. There are multiple spaces between the teeth. At the time of evaluation, existing restorations on teeth \#16 and 46 were defective resin composites, and teeth \#26 and 36 had large carious lesions.

The initial panoramic, bitewing and periapical radiographic examination (Figures 2 and 3), showed an early permanent dentition stage, with all permanent teeth present and covered by thin layer of enamel. The underlying dentin-pulp complex appeared normal. The dental age of this patient is earlier than his chronologic age. 

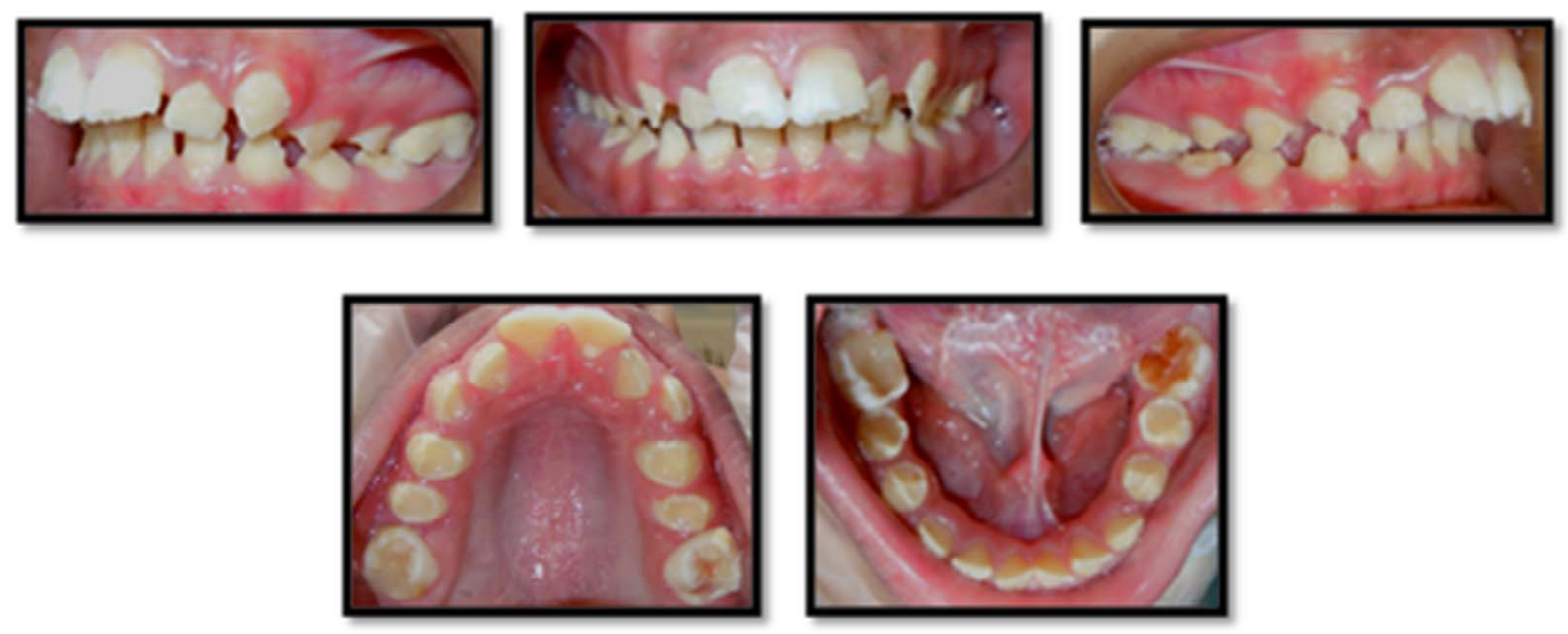

Figure 1. Preoperative clinical pictures of affected teeth.

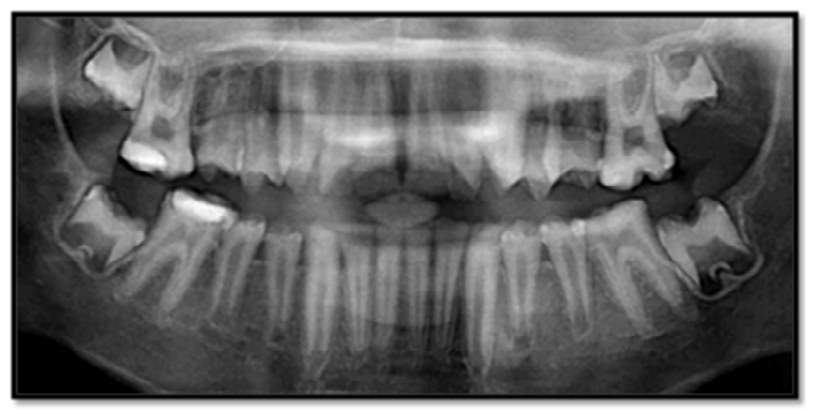

Figure 2. Panoramic view.
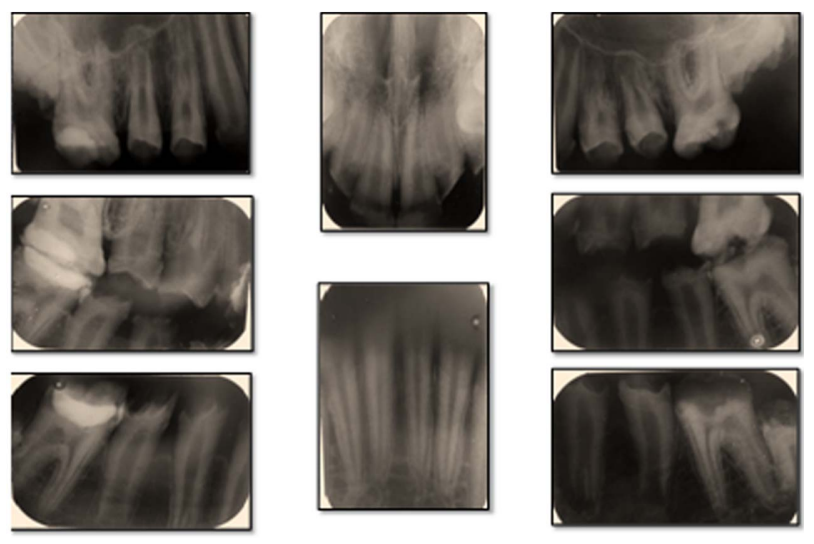

Figure 3. Periapical and bitewing view.

Tooth germs of all four third molars were absent.

\section{WHAT IS YOUR DIAGNOSIS}

\section{Discussion}

The clinical and radiographic findings suggested a hereditary enamel defect i.e. Amelogenesis Imperfecta (AI), and this diagnosis was strengthened by the positive fam- ily history of the same clinical presentation from the father side, his 8-year-old brother had suffered from the same condition, in addition, given history of disfigured primary dentition in the same manner. Consequently, based on the Witkop's classification [1,2], diagnosis of hypoplastic AI was made. Caries risk was assessed by interviewing the patient regarding diet, oral hygiene, and general lifestyle habits. The patient was placed by the assessment in a "high risk" category, given the presence of several predisposing factors, with AI carrying the most weight of all.

Amelogenesis imperfecta is a developmental inherited disturbance that is associated with malfunction of the enamel-forming proteins in the absence of a systemic disorder. In general, it affects all or nearly all of the teeth in both the primary and permanent dentitions $[3,4]$. The AI trait can be transmitted by either autosomal dominant, autosomal recessive, or X-linked modes of inheritance [5]. The estimated frequency of AI in the US population is 1:7000 [5]. The most widely accepted classification of AI includes three types: hypocalcified, hypoplastic and hypomaturation $[1,2,6]$. In the hypoplastic type, there is a deficiency in the quantity of enamel. The enamel is correctly mineralized and appears hard and shiny but is malformed. In the hypocalcified type, the enamel is formed in relatively normal amounts but is poorly mineralized, soft, and friable and can be easily removed from the dentin. In the hypomaturation type, abnormalities in the maturation stage of enamel formation result in a mottled appearance, opaque white to red-brown coloration, and enamel that is softer than normal and tends to chip from the underlying dentin [6]. To date, at least 14 forms of AI have been described based on the specific dental abnormalities and pattern of inheritance [1].

Children with AI can exhibit variable manifestations, 
with discoloration (yellow, brown, or gray), generalized areas of dentin exposed, pitted enamel with an increased susceptibility to plaque accumulation, caries, anterior open bite, and hypersensitivity to temperature changes as some of the most commonly occurring signs $[7,8]$.

The differential diagnosis of AI includes other forms of enamel dysmineralization which will exhibit a pattern based upon the time of insult, thus affecting the enamel forming at the time. In contrast, AI will affect all teeth similarly and can have a familial history. Fluorosis can mimic AI, but usually the teeth are not affected uniformly, often sparing the premolars and second permanent molars. A history of fluoride intake can aid in the diagnosis [8].

\section{THERAPY}

The complexity of the condition requires an interdisciplenary approach for optimal treatment outcomes. Different treatment options have been proposed in the dental literature. Options ranged from simple microabrasion [911], to composite restorations [12-15], gold or stainless steel crowns [13], all-ceramic crowns [7,16], metal-ceramic crowns $[7,12,17]$, and veneers $[18,19]$. Many case reports have focused on the early management of children and adolescents, and the use of bonded restorations has gained popularity because of the many benefits associated with these materials; excellent esthetics, conservative approach, and improved wear make their use advantageous [12,13].

Pediatric Dentistry and Prosthodontics participated in treatment planning. Regarding orthodontic therapy and achievement of a Class I occlusion, although needed, was not deemed a priority on the treatment sequence. The main goal of treatment was to provide transitional restorations for the protection of remaining tooth structure against further wear and sensitivity. Full coverage of all teeth with fixed prosthesis was identified as the ideal treatment; however, this approach requires the removal of a considerable amount of tooth structure. In addition, the patient's incomplete skeletal growth excluded this as an immediate option. Therefore, resin composites, particularly the indirect type, were chosen to provide an excellent prosthetic alternative until completion of skeletal growth. Indirect resin composites (also referred to as prosthetic composites) were introduced to dental practice to improve the clinical performance and provide optimal esthetics by ensuring precise marginal integrity, ideal proximal contacts and excellent anatomic morphology [20].

The following treatment sequence took place: Maxillary and mandibular arch impressions were made. Registration of skeletal relations was made with facebow and appropriate bite registration materials, and the case was mounted in a semi-adjustable articulator (Figure 4).

Evaluation of the articulated study models revealed that there is no need to increase the occlusal vertical dimension, because enough space is available for the thickness of restorative material of the posterior teeth. A diagnostic wax-up of all premolars, canines and incisors was made (Figure 5), followed by impression of each arch for construction of clear template material which has been used later on for temporization (Figure 6).

Stainless steel crowns were planned for coverage of defective teeth \# 16, 26, 36, and 46 in order to provide a posterior bite support, which will maintain the vertical dimension of occlusion. After cementation of the stainless steel crowns, all remaining permanent teeth were prepared for receiving the prosthetic composites. Tooth preparation involved axial and occlusal reduction limited to the removal of areas of thin hypoplastic enamel and undercuts (Figure 7).

Final maxillary and mandibular full arch impressions were made with a silicone impression material along with bite registration, which were sent to the dental laboratory for construction of prosthetic composite crowns. For temporization, the clear matrix template was filled with injectable temporization material (Protemp ${ }^{\mathrm{TM}}$ Plus, $3 \mathrm{M}$ ESPE). The matrix was carefully seated in place and kept in position through slight digital pressure for 1 - 3 minutes. Upon removal of the matrix, any areas of cervical overextension were trimmed, and any unsealed
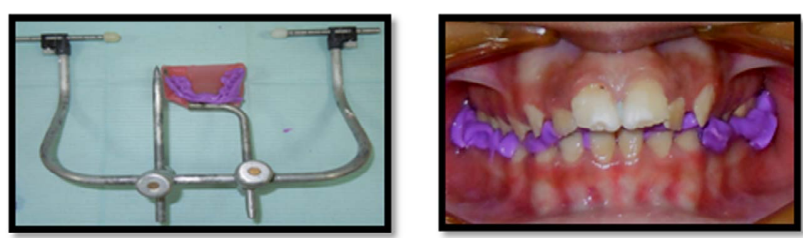

Figure 4. Registration of skeletal relations with face bow and bite registration.

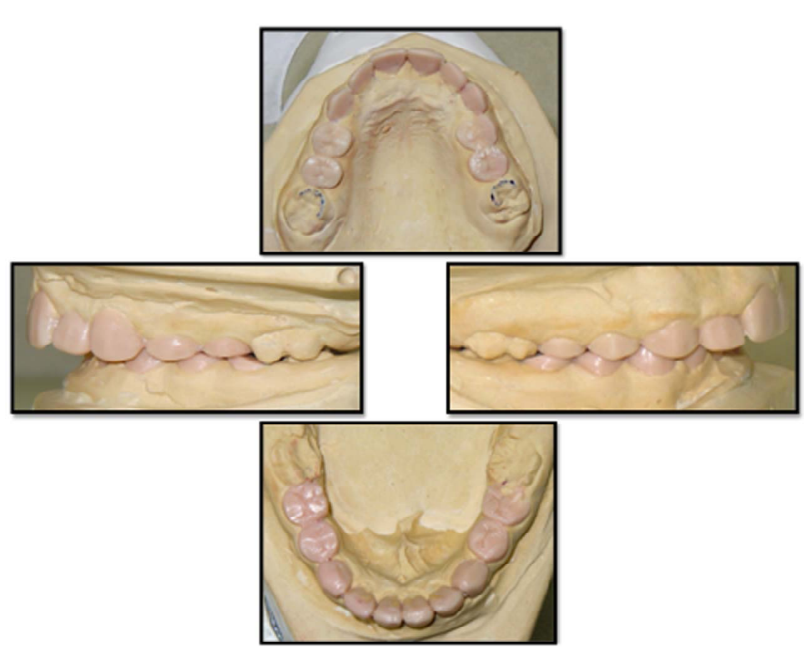

Figure 5. Diagnostic wax-up. 

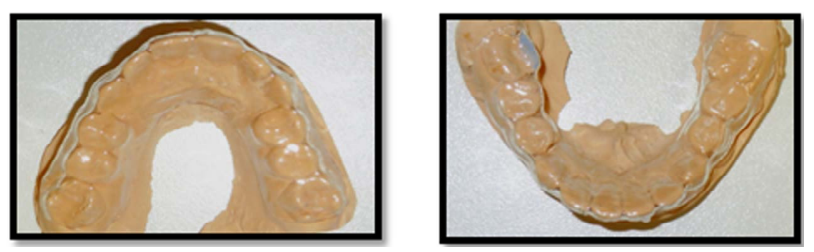

Figure 6. Clear template material for upper and lower arches.
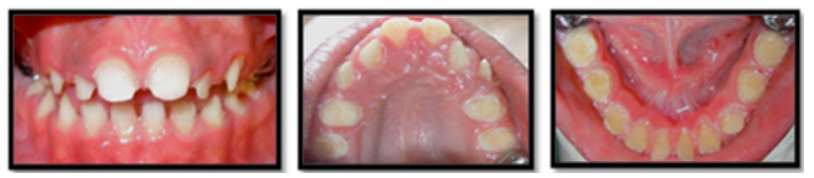

Figure 7. Teeth preparation.

areas along the margin were filled with a flowable resin. The temporary material was cemented with temporary cement (Figure 8).

Final composite crowns were delivered from the dental laboratory (Figure 9), and these crowns were tried-in the patient's mouth after cementing them with temporary cement. Occlusal and proximal contact, marginal integrity and anatomic morphology were checked. The final restorations were sent back to the dental laboratory for final polishing and glazing. Lastly, they were cemented with permanent resin cement (Figure 10). The final restorations exhibited not only good esthetics but also a return to an optimal masticatory function as well as providing complete coverage of all affected areas of the exposed dentin. These restorations not only addressed the patient's hypersensitivity issue but also contributed to the prevention of plaque accumulation.

\section{TREATMENT OUTCOME}

The use of composite restorative material resulted in successful and satisfactory enhancement of the patient's esthetic appearance, as well as protection against further wear and sensitivity thereby improving his functional demands. The longevity of the comprehensive treatment of this patient required strong emphasis on meticulous oral home-care regimen. Equally important to the success of this treatment are the periodic recall visits for monitoring of the restorations placed. The patient was monitored at 3-month intervals for 6 months. The restorations exhibited no signs of deterioration. The patient's oral hygiene was satisfactory. The restorations remained intact, with no discoloration, crazing, or carious lesions (Figure 11).

\section{CONCLUSIONS}

1) The importance of an interdisciplinary approach to the successful treatment of a patient with hypoplastic AI. has been concluded.

2) The conservativeness and suitability of full cover- age resin composite restorations for the transitional
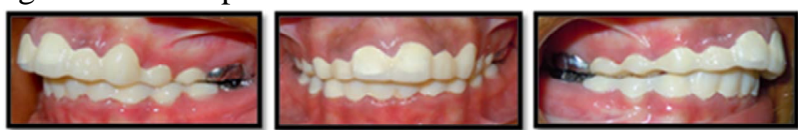

Figure 8. Temporization.
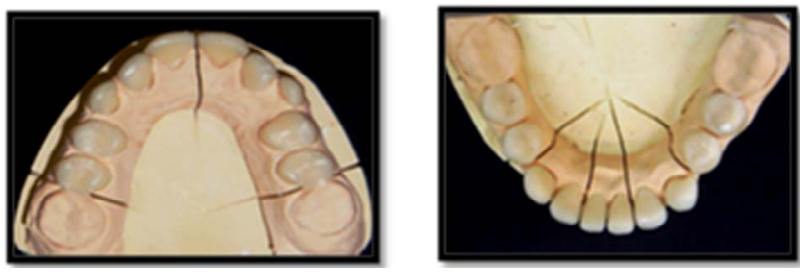

Figure 9. Final composite crowns.

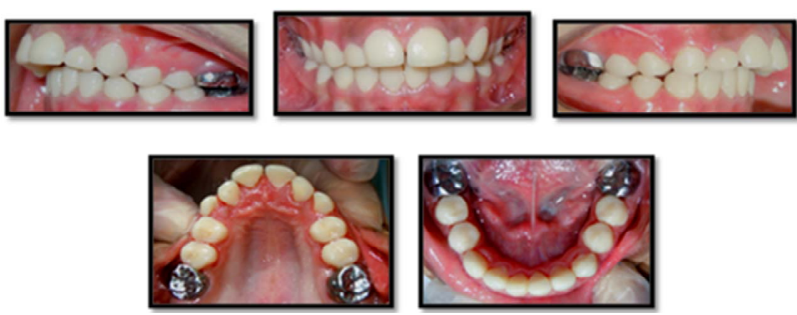

Figure 10. Permanent cementation with resin cement.
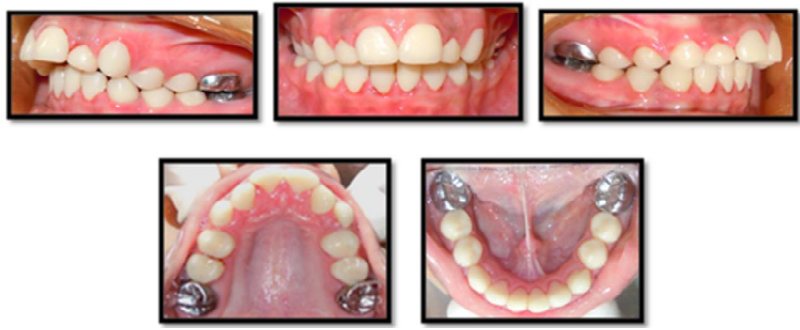

Figure 11. 6-month follow-up visit.

treatment of affected teeth on an adolescent patient who has not yet completed skeletal growth has also been concluded.

3) Treatment options vary considerably depending on several factors such as patient's age, socioeconomic status, severity of the disorder, and most importantly, the patient's cooperation. For optimal results, the treatment must be determined on an individual basis and with consultation of different disciplines.

4) This case emphasizes the importance of recognizing unusual pathology within the oral cavity, early diagnosis and prompt referral for subsequent management.

\section{REFERENCES}

[1] Witkop, C.J.J. (1988) Amelogenesis imperfecta, dentinogenesis imperfecta and dentin dysplasia revisited: Problems in classification. Journal of Oral Pathology \& Medicine, 17, 547-553.

http://dx.doi.org/10.1111/j.1600-0714.1988.tb01332.x 
[2] Crawford, P.J., Aldred, M. and Bloch-Zupan, A. (2007) Amelogenesis imperfecta. Orphanet Journal of Rare Diseases, 2, 17. http://dx.doi.org/10.1186/1750-1172-2-17

[3] Slayton, R. (2005) Congenital genetic disorders and syndromes, In: Pinkham, J.R., Casamassimo, P.S., Fields, H.W. Jr., McTigue, D.J., Nowak, A.J. Eds., Pediatric Dentistry: Infancy through Adolescence. 4th Edition, Elsevier Saunders, St. Louis, 257-274.

[4] Regezi, J., Sciubba, J. and Jordan, R. (2003) Oral pathology: Clinical-pathologic correlations. 4th Edition, WB Saunders, St. Louis, pp. 376-380.

[5] Backman, B. and Holmgren, G. (1988) Amelogenesis imperfecta: A genetic study. Human Heredity, 38, 189206. http://dx.doi.org/10.1159/000153785

[6] Akin, H., Tasveren, S. and Yeler, D.Y. (2007) Interdisciplinary approach to treating a patient with amelogenesis imperfecta: A clinical report. Journal of Esthetic and Restorative Dentistry, 19, 131-135. http://dx.doi.org/10.1111/j.1708-8240.2007.00083.x

[7] Gokce, K., Canpolat, C. and Ozel, E. (2007) Restoring function and esthetics in a patient with amelogenesis imperfecta: A case report. Journal of Contemporary Dental Practice, 8, 95-101.

[8] American Academy on Pediatric Dentistry Council on Clinical Affairs (2008) Guideline on oral heath care/dental management of heritable dental development anomalies. Pediatric Dentistry, 30, 196-201,

[9] Wong, F.S. and Winter, G.B. (2002) Effectiveness of microabrasion technique for improvement of dental aesthetics. Br Dent J, 193, 155-158. http://dx.doi.org/10.1038/sj.bdj.4801511

[10] Lynch, C.D. and McConnell, R.J. (2003) The use of microabrasion to remove discolored enamel: A clinical report. Journal of Prosthetic Dentistry, 90, 417-419. http://dx.doi.org/10.1016/S0022-3913(03)00503-1

[11] Rodd, H.D., Abdul-Karim, A., Yesudian, G., O’Mahony, J. and Marshman, Z. (2011) Seeking children's perspectives in the management of visible enamel defects. International Journal of Paediatric Dentistry, 21, 89-95. http://dx.doi.org/10.1111/j.1365-263X.2010.01096.x

[12] Markovic, D., Petrovic, B. and Peric, T. (2010) Case series: Clinical findings and oral rehabilitation of patients with amelogenesis imperfecta. European Archives of Paediatric Dentistry, 11, 201-208. http://dx.doi.org/10.1007/BF03262745

[13] Sabatini, C. and Guzman-Armstrong, S. (2009) A conservative treatment for amelogenesis imperfecta with direct resin composite restorations: A case report. Journal of Esthetic and Restorative Dentistry, 21, 161-169. http://dx.doi.org/10.1111/j.1708-8240.2009.00258.x

[14] Agackiran, E., Tumen, E.C., Celenk, S., Bolgul, B. and Atakul, F. (2011) Restoring aesthetics and function in a young boy with hypomature amelogenesis imperfecta: A case report. ISRN Dentistry, 2011, 586854.

[15] Sockalingam, S. (2011) Dental rehabilitation of amelogenesis imperfecta using thermoformed templates. Journal of Indian Society of Pedodontics and Preventive Dentistry, 29, 53-56. http://dx.doi.org/10.4103/0970-4388.79938

[16] Gisler, V., Enkling, N., Zix, J., Kim, K., Kellerhoff, N.M. and Mericske-Stern, R. (2010) A multidisciplinary approach to the functional and esthetic rehabilitation of amelogenesis imperfecta and open bite deformity: A case report. Journal of Esthetic and Restorative Dentistry, 22, 282-293. http://dx.doi.org/10.1111/j.1708-8240.2010.00354.x

[17] Bharath Shetty, Y. and Shetty, A. (2010) Oral rehabilitation of a young adult with amelogenesis imperfecta: A clinical report. Journal of Indian Prosthodontic Society, 10, 240-245. http://dx.doi.org/10.1007/s13191-010-0037-6

[18] Brignall, I., Mehta, S.B., Banerji, S. and Millar, B.J. (2011) Aesthetic composite veneers for an adult patient with amelogenesis imperfecta: A case report. Dent Update, 38, 594-603.

[19] Ramos, A.L., Pascotto, R.C., Iwaki Filho, L., Hayacibara, R.M. and Boselli, G. (2011) Interdisciplinary treatment for a patient with open-bite malocclusion and amelogenesis imperfecta. American Journal of Orthodontics and Dentofacial Orthopedics, 139, S145-153. http://dx.doi.org/10.1016/j.ajodo.2009.05.031

[20] Nandini, S. (2010) Indirect resin composites. Journal of Conservative Dentistry, 13, 184-194. http://dx.doi.org/10.4103/0972-0707.73377 